\title{
Uso del Plasma Rico en Plaquetas para el tratamiento de las úlceras de miembro inferior. Estudio piloto
}

\author{
Ma José de la Torre Barbero ${ }^{1}$, Ma José Estepa Luna ${ }^{1}$, José Rubio Moreno ${ }^{1}$ \\ ${ }^{1}$ Hospital Universitario Reina Sofía. Córdoba. España
}

\section{Correspondencia}

Ma José de la Torre Barbero

Correo electrónico: torrebarbero@gmail.com

Recibido: 10/05/2020

Aceptado: 09/06/2020

\section{RESUMEN}

Cada vez hay más pruebas que sugieren que la cicatrización de heridas en las úlceras crónicas vasculares y del pie diabético depende del factor de crecimiento y que el suministro terapéutico de estos factores de crecimiento a las heridas por vía tópica tiene la capacidad potencial de acelerar la cicatrización de las heridas junto con el cuidado convencional de las heridas

Se ha diseñado y proyectado un estudio prospectivo cuasiexperimental con el objetivo de evaluar la seguridad y eficacia del gel de plasma autólogo rico en plaquetas para el tratamiento de las úlceras vasculares de miembros inferiores y de pie diabético con retraso de la cicatrización. Mediante este estudio piloto se ha valorado la viabilidad de dicho estudio y la idoneidad del protocolo de actuación, de las variables recogidas y del instrumento de medida.

Este estudio piloto fue realizado entre septiembre de 2018 y marzo de 2020 en el Servicio de Cirugía Vascular del Hospital Universitario Reina Sofía de Córdoba. Participaron pacientes, con úlceras vasculares o de pie diabético que presentaban retraso en el proceso de cicatrización y a los que se les aplicó PRP

En el estudio piloto han participado cuatro pacientes con una media de edad 62,25 años. El $100 \%$ fueron hombres con lesiones de pie diabético. El 50\% presentaba arteriopatía periférica asociada, con una media en el retraso de la cicatrización de 17 meses con intervalo de 12-20. El tiempo medio de cicatrización de las úlceras fue de 7,6 semanas. En ningún caso se detectaron efectos adversos relacionados con la aplicación de PRP.

Según estos resultados preliminares, la aplicación de PRP sobre el lecho de las heridas vasculares y de pie diabético que no cicatrizan es una opción terapéutica segura y eficaz.

\section{PALABRAS CLAVE}

plasma rico en plaquetas; factores de crecimiento; úlceras pierna; pie diabético; cicatrización de las heridas.

\section{SUMMARY}

There is increasing evidence to suggest that wound healing in chronic vascular and diabetic foot ulcers is dependent on growth factor and that the therapeutic delivery of these growth factors to topical wounds has the potential ability to accelerate healing. of wounds together with conventional wound care

A prospective quasi-experimental study has been designed and planned with the objective of evaluating the safety and efficacy of platelet-rich autologous plasma gel for the treatment of vascular ulcers of the lower limbs and diabetic foot with delayed healing. Through this pilot study, the viability of said study and the suitability of the action protocol, the variables collected and the measuring instrument have been assessed.

This pilot study was carried out between September 2018 and March 2020 at the Vascular Surgery Service of the Reina Sofía University Hospital in Córdoba. Participated patients with vascular or diabetic foot ulcers who had delayed healing process and to whom PRP was applied

Four patients with a mean age of 62.25 years participated in the pilot study. $100 \%$ were men with diabetic foot injuries. 50\% had associated peripheral artery disease, with a mean healing delay of 17 months with an interval of $12-$ 20. The mean healing time of the ulcers was 7.6 weeks. In no case were any adverse effects related to the application of PRP detected.

Based on these preliminary results, the application of PRP to the bed of non-healing vascular and diabetic foot wounds is a safe and effective therapeutic option.

\section{KEYWORDS}

platelet rich plasma; growth factors, leg ulcer; diabetic foot ulcer; wound healing. 


\section{Introducción}

Hoy día, a pesar de los avances del conocimiento de las ciencias de la salud y de la evolución del sistema sanitario, las heridas continúan siendo un importante problema de salud pública, tanto en España como en otros países de nuestro entorno, que afecta a pacientes de todos los niveles asistenciales (1).

Las heridas crónicas (HC) constituyen uno de los más importantes problemas asistenciales que afectan al sistema de salud por la disminución de la calidad de vida de quien la padece y de sus cuidadores, su elevado coste económico y por la dedicación de mucho tiempo de los profesionales de enfermería (2). La cicatrización de dichas heridas es un fenómeno complejo, en muchos casos no conocido en su totalidad, que exige los conocimientos específicos y el trabajo de un equipo multidisciplinar para poder abarcar todos los aspectos y necesidades.

Las HC no siguen un proceso de reparación normal y se pueden estancar en alguna fase de cicatrización ocasionando el impedimento de restauración integra del tejido, con una cicatrización más pausada, pese a proporcionarles tratamiento adecuado.

Las úlceras vasculares (arteriales o venosas) presentan una elevada incidencia entre población, y tienen importantes repercusiones socioeconómicas y sanitarias. El aumento de la esperanza de vida ha supuesto un aumento de la prevalencia e incidencia de estas lesiones, con una afectación de la calidad de vida y pérdida de autonomía de quienes las padecen. Según Conferencia Nacional de Consenso sobre las Úlceras de la Extremidad Inferior (C.O.N.U.E.I.) (3), la Incidencia es de 3 a 5 nuevos casos por mil personas y año y la prevalencia de 0,10 a $0,30 \%$. Ambos datos, deben multiplicarse por dos cuando se considere el segmento de población de edad > a 65 años.

Las úlceras del pie diabético, son una de las complicaciones más significativas de la diabetes y se manifiestan hasta en un $15 \%$ de los pacientes diagnosticados, siendo una de las causas más habituales de amputación de la extremidad inferior en Europa y Estados Unidos (4). El riesgo de una amputación se relaciona con la fisiopatología compleja de la neuropatía y de la isquemia en el pie del diabético, lo cual hace imposible que el pie permanezca intacto bajo el estrés de la vida diaria (5)

En los últimos años se han producido importantes avances en el campo del tratamiento de las úlceras, así como en el concepto de la úlcera crónica como un proceso inflamatorio con implicación de citoquinas proinflamatorias y déficit de factores de crecimiento (6). El plasma rico en plaquetas es un producto derivado de la sangre, rico en péptidos y proteínas de señalización intercelular, así como en citoquinas capaces de intervenir en cada una de las etapas de la regeneración de varios tejidos. Principalmente se le han atribuido efectos antiinflamatorios en diferentes lesiones, así como otros efectos biológicos sobre las células y tejidos (7).
La definición del PRP indica al menos 2,5-1000 x103 plaquetas/ $\mu \mathrm{L}$ suspendidas en plasma (de 2 a 7 veces el valor basal); menor concentración no tendrá efecto y más concentración no incrementará la respuesta biológica, o bien, podría tener efectos inhibitorios en la regeneración de heridas y disminuir la angiogénesis (8).

De acuerdo con sus aplicaciones, existen tres formulaciones terapéuticas básicas: en presentación líquida, usado como gotas, principalmente utilizado a nivel oftalmológico y cirugías de implantes dentales; en forma gelificada, usado para lesiones en piel, o bien, en forma de una membrana, es decir, con más cuerpo y densidad, apropiada para tratar lesiones de tejidos blandos y sellar heridas quirúrgicas (9). Existen numerosos métodos disponibles para separar PRP de la sangre total. Sin embargo, al considerar las acciones potenciales de PRP, se requiere una gran precaución porque la composición de PRP puede variar mucho debido a las variables en el proceso de preparación (10) (11).

EI PRP induce la regeneración de heridas y la reparación de tejidos y una prematura cicatrización y reparación de las mismas, promoviendo la síntesis de fibroblastos y osteoblastos; aumenta la revascularización mediante la liberación de factores de crecimiento, los cuales son los iniciadores universales de la cicatrización (12). Técnicamente, al ser un producto autólogo con poca manipulación, es seguro, y el riesgo de adquirir una enfermedad infectocontagiosa es bastante bajo. También hay estudios que señalan que la aplicación del PRP reduce considerablemente el dolor. La primera respuesta ante una lesión es la inflamación, acompañada de dolor neuropático, debido a la hiperexcitabilidad de las neuronas nociceptivas. Una vez suprimida la inflamación, disminuye el estímulo a los nociceptores y con ello, el decremento del dolor (10). Esto incide enormemente en el estado de ánimo y en la calidad de vida del paciente.

Cada vez hay más pruebas que sugieren que la cicatrización de heridas en las úlceras crónicas vasculares y del pie diabético depende del factor de crecimiento y que el suministro terapéutico de estos factores de crecimiento a las heridas por vía tópica tiene la capacidad potencial de acelerar la cicatrización de las heridas junto con el cuidado convencional de las heridas (13). En la actualidad, no existe un protocolo estándar de producción o preparación, tampoco se ha descrito una dosis específica; la forma de aplicación es muy variable y depende de la condición por tratar. Existen muchos métodos para obtener PRP y procesar ese plasma en un producto para la aplicación tópica de heridas. Todos implican el uso de una centrífuga para fraccionar la sangre, separando los glóbulos rojos del plasma rico en plaquetas. Las diferencias importantes en los métodos de centrifugación implican la velocidad, la velocidad, la aceleración, la desaceleración y la angulación y el radio del rotor de la centrífuga (14-16). Otras diferencias en los métodos de procesamiento del plasma implican la activación de las plaquetas mediante la adición de 
varios tipos de trombina, trombina con cloruro de calcio, cloruro de calcio solo o baproxobina al PRP y utilizando un método de congelación y descongelación que lisa las plaquetas para acceder al contenido (16).

Se ha diseñado y proyectado un estudio prospectivo cuasiexperimental con el objetivo de evaluar la seguridad y eficacia del gel de plasma autólogo rico en plaquetas para el tratamiento de las úlceras vasculares de miembros inferiores y de pie diabético con retraso de la cicatrización. Mediante este estudio piloto se ha valorado la viabilidad de dicho estudio y la idoneidad del protocolo de actuación, de las variables recogidas y del instrumento de medida.

\section{Metodología}

Este estudio piloto fue realizado entre septiembre de 2018 y marzo de 2020 en el Servicio de Cirugía Vascular del Hospital Universitario Reina Sofía de Córdoba. Participaron pacientes mayores de edad, de ambos sexos, con úlceras vasculares o de pie diabético que presentaban retraso en el proceso de cicatrización y a los que se les aplicó PRP.

Los criterios de inclusión fueron: pacientes mayores de edad, de ambos sexos, que hubiesen dado su consentimiento para participar en el estudio y que presentasen úlceras vasculares o de pie diabético de más de 6 meses de evolución y que no cicatrizaban a pesar de haber recibido un abordaje adecuado basado en la valoración integral de las necesidades de cuidados del paciente y en las características de las lesiones: presencia o no de infección, viabilidad de los tejidos, grado de exudado y necesidad de descargas o técnicas avanzadas como terapia de presión negativa o apósito moduladores de metaloproteasas.

Los criterios de exclusión fueron: pacientes con isquemia crítica (Grados III-IV de Fontaine) no revascularizados, presencia de infección y/o osteomielitis (se realizaba una radiografía a todos los pacientes que presentasen un Probe to bone positivo), pacientes oncológicos (en tratamiento con quimioterapia o radioterapia) y pacientes terminales.

Las variables recogidas fueron: edad, sexo, etiología de la herida, tiempo de evolución, superficie de la herida (en $\mathrm{cm}^{2}$ ), cicatrización (sí o no), tiempo (en semanas) hasta la epitelización completa desde que se inicia la aplicación de PRP y efectos adversos reportados por el paciente o detectados por los investigadores.

Tanto la captación de pacientes como la recogida de datos y la aplicación del tratamiento fueron realizados por los autores de este artículo.

El protocolo de actuación seguido en este estudio fue:

Extracción de sangre. Se procede a la extracción de $30 \mathrm{cc}$ de sangre con $3 \mathrm{ml}$ de citrato sódico como anticoagulante.

Separación del suero y obtención del coágulo: La sangre se procesa inmediatamente, centrifugándola a $2800 \mathrm{rpm}$ durante 5 minutos a temperatura ambiente en una centrifugadora. El plasma obtenido se separa en
2 fracciones. La fracción superior de aproximadamente 6-7 cc se denomina plasma pobre en factores de crecimiento (PPP) y se desecha. La fracción inferior de aproximadamente $7-8 \mathrm{cc}$, se conoce como plasma rico en factores de crecimiento o plasma autólogo rico en plaquetas (PRP), que es la que utilizaremos para preparar el coágulo. Para obtener una textura gelificada, añadimos cloruro de cálcico e introducimos en estufa a 38ㅇ C.

Aplicación en la herida: Colocación del coágulo de plaquetas en el lecho de la úlcera, cubriéndolo con un apósito de silicona y apósito de alginato para la gestión del exudado del exudado. La cura se mantiene durante una semana.

El protocolo seguido en este estudio consiste en una valoración general del paciente; examen de heridas; exámenes diagnósticos instrumentales, microbiológicos si se precisan; y adquisición de imágenes fotográficas con seguimiento semanal.

Previo a la aplicación del PRP, se realizó medición del área de las úlceras en la primera evaluación, que se repitió en cada una de la visitas, comparando dichas áreas al inicio del tratamiento, a las 3 semanas y a las 10 semanas o finalizado el proceso de cicatrización.

Para la medición de las úlceras se utilizaron reglas de papel desechables, midiéndose el largo y el ancho en centímetros y calculando el área (largo $x$ ancho) en $\mathrm{cm}^{2}$. Para evitar la variabilidad, la medición de las úlceras se realizó siguiendo el método estándar descrito por Sussman (17).

Previo a la aplicación del PRP sobre la superficie de las úlceras se realizaba un lavado de la superficie con suero fisiológico con técnica de asepsia y, si era necesario, se realizaba un desbridamiento para la retirada del tejido no viable del lecho y los bordes perilesionales.

Posteriormente se protegían los bordes perilesionales con una crema barrera de óxido de zinc, se aplicaba el gel de PRP adaptándolo al lecho de la lesión y se sujetaba con una malla de silicona y un apósito secundario para la gestión del exudado. La aplicación del PRP se realizó cada 7 días.

Este estudio se ha realizado en base a los principios de la Declaración de Helsinki y a la legislación nacional vigente. Se solicitó el consentimiento informado firmado a todos los participantes.

Se proporcionó información verbal del tratamiento y procedimiento a cada paciente para conseguir la máxima colaboración del paciente en el posicionamiento y la realización del procedimiento.

\section{Resultados}

En este estudio piloto han participado cuatro pacientes con una media de edad 62,25 años con un mínimo 56 y un máximo de 71 años. El 100\% fueron hombres que presentaban lesiones de pie diabético. El $50 \%$ presentaba arteriopatía periférica asociada, con una media en el retraso de la cicatrización de 17 meses 
con intervalo de 12-20. El tiempo medio de cicatrización de las úlceras fue de 7,6 semanas con un intervalo de 5-10. A un paciente se le suspendió el tratamiento a las 5 semanas por el estado de alarma decretado por la pandemia de Covid 19. En ningún caso se detectaron efectos adversos relacionados con la aplicación de PRP.

\section{Caso 1}

Paciente varón de 56 años con antecedentes de trastorno de ansiedad, polineuropatía diabética; hepatopatía crónica enólica, retinopatía diabética; HTA; lumbalgia; diabetes méllitus tipo 2 .

El paciente presentaba úlcera neuropática en borde externo del talón del pie derecho de 12 meses de evolución. Dada la mala evolución de la úlcera, que presentaba lecho con tejido de granulación pálido y friable y bordes hiperqueratósicos, se decide, con el consentimiento del paciente, iniciar tratamiento con PRP. Se programa cura semana y se aplica el protocolo de cura establecido para esta técnica.

Al inicio del tratamiento el área de la úlcera era $9 \mathrm{~cm}^{2}$, a las 3 semanas el área era de $7,5 \mathrm{~cm}^{2}$, con una reducción de la superficie de la lesión de un $17 \%$ en ese periodo. Se consigue la epitelización completa de la lesión en 8 semanas. En la figura 1 se muestran las imágenes al inicio del tratamiento y tras la cicatrización completa.
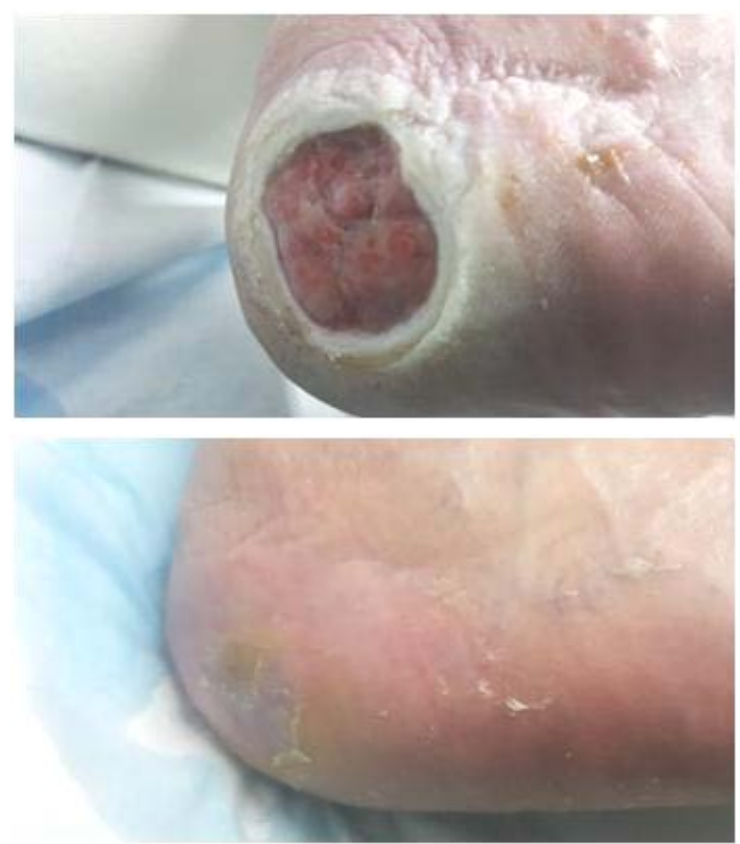

Figura 1. Imágenes al inicio del tratamiento y tras la cicatrización completa a las 8 semanas

\section{Caso 2}

Paciente varón de 62 años con antecedentes de anemia megaloblástica, diabetes mellitus tipo 2 , obesidad y arteriopatía periférica, que presenta amputación transmetatarsiana en pie derecho producida infección grave de pie diabético de 20 meses de evolución. Ante el retraso en la cicatrización se decide con el consentimiento del paciente iniciar tratamiento con PRP.

Al inicio del tratamiento el área de la lesión era de $4,5 \mathrm{~cm}^{2}$, a las 3 semanas de tratamiento el área de la úlcera había disminuido un $12 \%$ (medía $4 \mathrm{~cm}^{2}$ ). A las 5 semanas se suspende el tratamiento por la pandemia de Covid 19, presentando la lesión una superficie de 2,5 $\mathrm{cm}^{2}$. En la figura 2 se muestran las imágenes al inicio del tratamiento y a las 5 semanas cuando se suspende el tratamiento.
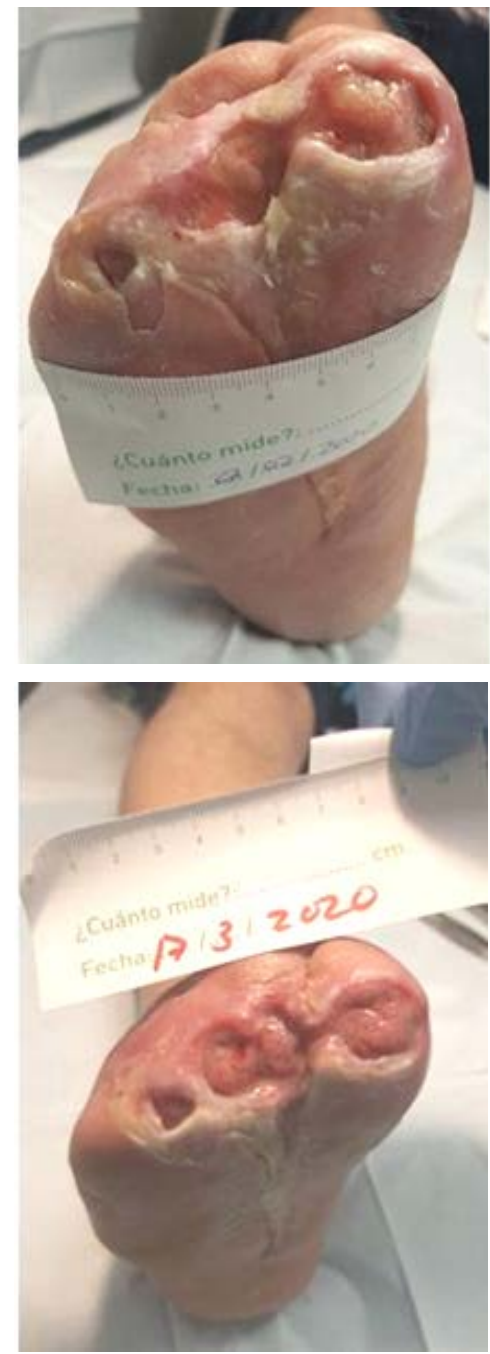

Figura. 2. imágenes al inicio del tratamiento y a las 5 semanas cuando se suspende el tratamiento

\section{Caso 3}

Paciente varón de 71 años con antecedentes de dislipemia, retinopatía diabética proliferativa y anemia.

Presentaba úlcera neuropática en talón derecho que cuando acude a nuestra consulta llevaba 7 meses de evolución. Tras 17 meses de seguimiento en el que se realizan varios tipos de tratamiento sin conseguir una evolución favorable de la lesión, se decide iniciar tratamiento con PRP.

Al inicio del tratamiento la lesión tenía un área de $10 \mathrm{~cm}^{2}$, a las 3 semanas de tratamiento el área de la 
lesión había disminuido un $30 \%$ (medía $7 \mathrm{~cm}^{2}$ ), y se consiguió la epitelización completa de la lesión en 10 semanas. En la figura 3 se muestran las imágenes al inicio del tratamiento y tras la cicatrización completa.
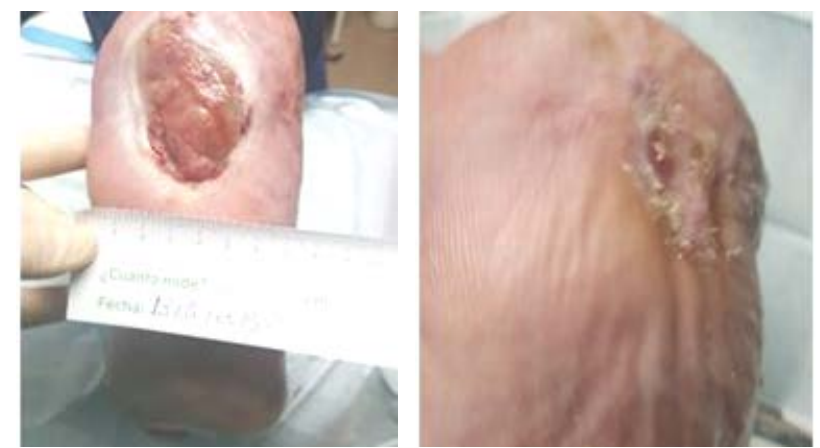

Figura. 3. Imágenes al inicio del tratamiento y tras la cicatrización completa a las 10 semanas

\section{Caso 4}

Paciente varón de 60 años con antecedentes de síndrome metabólico, ictus isquémico, miocrdiopatía dilatada, hipertensión arterial, hiperuricemia, diabetes mellitus, adenocarcinoma de próstata, depresión, arteriopatía periférica y amputación del 1o dedo del pie derecho.

El paciente presentaba úlcera en la cara plantar del pie izquierdo, sobre la cabeza del primer metatarsiano de 18 meses de evolución. Tras la aplicación de diversos tipos de tratamiento y el estancamiento de la lesión seguía estancada. se inicia tratamiento con PRP. Al inicio del tratamiento con PRP el área de la úlcera era 2 $\mathrm{cm}^{2}$, a las 3 semanas la lesión tenía una superficie de $0,70 \mathrm{~cm}^{2}$, lo que supone una reducción del área de un $65 \%$ en ese periodo. La epitelización completa se consiguió a las 5 semanas.

En la figura 4 se muestran las imágenes al inicio del tratamiento y tras la cicatrización completa a las 5 semanas.
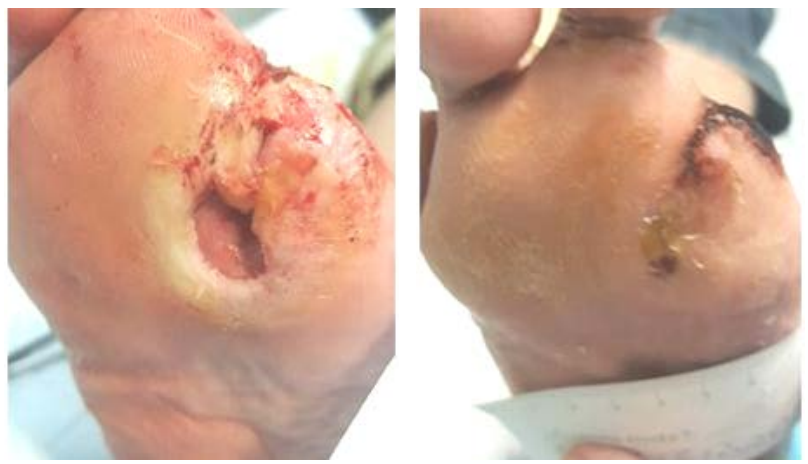

Figura. 4. Imágenes al inicio del tratamiento y tras la cicatrización completa a las 5 semanas

No se observaron efectos secundarios sistémicos o en el sitio de la herida. Ocasionalmente, se observó maceración alrededor de la piel perilesional.

\section{Discusión}

La aplicación del plasma rico en plaquetas es cada día más utilizada en la práctica clínica en diversas especialidades para lograr una aceleración en los procesos de regeneración tisular (18). Sin embargo al existir diversos métodos de obtención del plasma, las preparaciones varían en las concentraciones de plaquetas y la consistencia del gel resultante. Varios autores han declarado que gran parte de la variabilidad reportada en los resultados de curación de heridas con el uso de PRP se deba, probablemente a la diversidad de dispositivos, métodos y estrategias clínicas utilizadas para obtener y aplicar productos derivados de PRP. (19-21).

Los resultados obtenidos en este estudio piloto, nos llevan a considerar, que el plasma rico en factor de crecimiento plaquetario, es una opción en el manejo y cicatrización de las úlceras vasculares y de pie diabético. Otros autores (22) también proponen la terapia con PRP, como una alternativa de tratamiento, sobre todo en casos de úlceras cutáneas crónicas donde los tratamientos convencionales resulten improductivos. EI estudio de Enríquez-Vega 2010 (5), concluye que el factor de crecimiento contenido en el plasma rico en plaquetas es una alternativa terapéutica autóloga e inocua que mostró una eficacia de $79.2 \%$ en la cicatrización de úlceras isquémicas en pie diabético.

Nuestros resultados muestran una reducción del área de las úlceras después de 3 semanas. En el estudio de Cervelli 2010, también obtienen similares resultados a las 3 semanas de evolución, además encontraron que la curación se producía en menos de 6 semanas en el $47 \%$ de los pacientes y la curación completa de heridas en el $57 \%$ de los pacientes en 3 meses (23).

Los resultados de este estudio, se encuentran en línea con los estudios encontrados en la literatura, siendo en nuestra serie de casos el tiempo medio de curación inferior a la cura con tratamiento convencionales, Un estudio en pacientes con úlceras isquémicas con pie diabético, manejadas con tratamiento convencional, encontró que de media el $41 \%$ de las úlceras, cicatrizaban a las 12 semanas (16); en otros ensayo clínico se encontró que las úlceras tardaban un promedio de 91 días en cicatrizar con terapia convencional (24).

El hecho de no contar con un grupo control implica limitaciones a la hora de interpretar los datos. Sin embargo, es evidente una reducción importante en el tiempo de curación del paciente; teniendo en cuenta que muchos de ellos habían sido tratados previamente sin lograr dicha curación a lo largo de muchos meses. Las úlceras, tras la aplicación del tratamiento con PRP han cicatrizado en un tiempo medio de aproximadamente 7 semanas. En el estudio de Monton Echevarria 2007 (18), la media de tiempo de cicatrización de las lesiones fue de alrededor de los 3 meses. 


\section{Conclusión}

Los resultados de este estudio piloto muestran la viabilidad y pertinencia del estudio de investigación proyectado para intentar valorar la capacidad del PRP para regenerar el tejido y conseguir una reducción en el tiempo necesario para la completa epitelización y cierre de las heridas vasculares y de pie diabético refractario a los tratamientos convencionales. Según estos resultados preliminares, la aplicación de PRP sobre el lecho de las heridas vasculares y de pie diabético que no cicatrizan es una opción terapéutica segura y eficaz.

Sería necesario realizar ensayos clínicos con grupo control que permitan comprobar la eficacia, seguridad y eficiencia de la aplicación de PRP en todo tipo de heridas crónicas, así como comparar los resultados de dicha terapia con los resultados que se obtienen mediante terapias convencionales.

\section{Bibliografía}

1. Dato Vidal M M. Plasma rico en plaquetas en el tratamiento de las heridas crónicas. Documento en internet. Disponible en: https://digitum.um.es/ digitum/bitstream/10201/56914/1/TFG\%20Mar\%C 3\%ADa\%20de\%20las\%20Mercedes\%20Dato\%20Vid al-1.pdf.

2. Lorenzo Hernández MP, Hernández Cano RM, Soria Suárez, MI. Heridas crónicas atendidas en un servicio de urgencias. Enfermería Global.2014; 35:23-31.

3. Marinel.lo Roura J, Verdú Soriano J (Coord.). Conferencia nacional de consenso sobre las úlceras de la extremidad inferior (C.O.N.U.E.I.). Documento de consenso 2018. 2a ed. Madrid: Ergon; 2018.

4. Esperón Güimil JA, Loureiro Rodríguez MT, Antón Fuentes V, Rosendo Fernández JM, Pérez García I., Soldevilla-Agreda JJ. Variabilidad en el abordaje de las heridas crónicas: ¿qué opinan las enfermeras? Gerokomos. 2014; 25(4):171-177.

5. Enríquez-Vega M E, Bobadilla-Flores NO, RodríguezJiménez OA, Guerra-Márquez A, Carrasco-Nava L, Varela-Silva J. Plasma rico en plaquetas para el tratamiento de úlceras isquémicas del paciente diabético. Revista Mexicana de ANGIOLOGIA. 2012; 40 (2): 51-56.

6. Gómez Sánchez ME, Azaña Defez JM, Escario Travesedo E, Gómez Bajo GJ, López Villaescusa MT, Martínez Martínez ML et al. Concentrado de plasma rico en plaquetas: revisión y uso en úlceras de larga evolución. Med Cutan Iber Lat Am 2015; 43 (2): 125-131.

7. Castro-Piedra SE, Arias-Varela KA. Actualización en plasma rico en plaquetas. Acta méd costarric 2019; 61 (4):142-151.

8. Castro-Piedra S E, Arias Varela K A. Actualización en plasma rico en plaquetas. Acta méd. costarric.2019; 61 (4):142-151.
9. Chícharo D, Rubio M, Damiá E, Carrillo J, Cuervo B, Peláez P. Platelet Rich Plasma: New Insights for Cutaneous Wound Healing Management. J FunctBiomater. 2018. 9: 1-20.

10. Kuffler D. Platelet Rich Plasma Promotes Axon Regeneration, Wound Healing and Pain Reduction: Fact or Fiction. Mol Neurobiol. 2015. 52:990-1014.

11. Burnouf T, Goubran HA, Chen TM, Ou KL, El-Ekiaby $M$, Radosevic $M$. Blood-derived biomaterials and platelet growth factors in regenerative medicine. Blood Rev 2013; 27:77

12. Olmedo Gallardo AK, Miranda Muñoz GC, Layana Varas CM, Tettamanti Miranda D, Varas Diminich P. Plasma autólogo enriquecido en plaquetas vs debridaciones continuas en úlceras de miembros inferiores: estudio controlado en pacientes diabéticos e insuficientes venosos que acuden al Hospital Teodoro Maldonado Carbo en el período 2010. [Internet]. 2010. Disponible en: http://repositorio.ucsg.edu.ec/bitstream/3317/625/ 1/T-UCSG-PRE-MED-90.pdf [consulta: 20 jun 2020].

13. Ayobami Akingboye A, Giddins S,Gamston P, Tucker A, Navsaria H; Kyriakides C. Application of Autologous Derived-Platelet Rich Plasma Gel in the Treatment of Chronic Wound Ulcer: Diabetic Foot Ulcer. JECT. 2010; 42:20-29.

14. Roukis TS, Zgonis T, Tiernan B. Autologous plateletrich plasma for wound and osseous healing: a review of the literature and commercially available products. Adv Ther. 2006;23(2):218-237.

15. Arora NS, Ramanayake T, Ren YF, Romanos GE. Platelet-rich plasma: a literature review. Implant Dent. 2009;18(4):303-310.

16. Frykberg RG, Driver VR, Carman D, Lucero B, BorrisHale C, Carelyn P. Chronic Wounds Treated With a Physiologically Relevant Concentration of Plateletrich Plasma Gel: A Prospective Case Series. Ostomy Wound Management 2010;56(6):36-44.

17. Sussman C. Wound measurements. In: Sussman C, Bates-Jensen B (eds). Wound Care: A Collaborative Practice Manual for Physical Therapists and Nurses. Gaithersburg, MD: Aspen; 2001:120-141

18. Montón Echeverría J, Pérez Redondo S, Gómez Bajo GJ. Experiencia clínica en el empleo de factores de crecimiento autólogos obtenidos de plasma rico en plaquetas Resumen Abstract Clinical experience related to the use of autologous platelet rich plasma. Cir.plást. iberolatinoam 2007; .33 (3):155162.

19. Eppley BL, Woodell JE, Higgins J. Platelet quantification and growth factor analysis from platelet-rich plasma: implications for wound healing. Plast Reconstr Surg. 2004; 114(6)15021508. 
20. Ficarelli E, Bernuzzi G, Tognetti E, et al. Treatment of chronic venous leg ulcers by platelet gel. Dermatol Ther. 2008; 21(1):13-17.

21. Smith S, Roukis T. Bone and wound healing augmentation with plateletrich plasma. Clin Podiatr Med Surg. 2009; 26(4):559-588.)

22. Calderón Ibáñez T. La importancia de la cronicidad de las úlceras cutáneas; terapia alternativa con plasma rico en plaquetas. TFG, documento en internet. Disponible en: https://repositorio.unican.es/xmlui/ bitstream/handle/10902/11696/Calder\%c3\%b3n\%20
Ib\%c3\%a1\%c3\%b1ez\%20Tamara.pdf?sequence=4\&is Allowed $=y$

23. Cervelli $V$ et al. Tissue Regeneration in Loss of Substance on the Lower Limbs through Use of Platelet-Rich Plasma, Stem Cells from Adipose Tissue, and Hyaluronic Acid. Advances in Skin \& Wound Care 2010; 23 (6):262-272.

24. Castro G, Liceaga G, Arrioja A, Calleja JM, Espejel A. Guía Clínica basada en evidencia para el manejo del paciente diabético. Med Int Mex 2009; 25(6): 481526. 